\title{
IgG4-Related Sclerosing Mesenteritis
}

\author{
Seok Joo Lee · Cheol Keun Park · Woo Ick Yang · Sang Kyum Kim \\ Department of Pathology, Yonsei University Medical Center, Seoul, Korea
}

Sclerosing mesenteritis (SM) is a rare disease first described by Sulla ${ }^{1}$ in 1924 under the name "retractile mesenteritis." SM shows fibrosis with fat necrosis and chronic inflammation on microscopic examination. ${ }^{2}$ In particular, if the histologic and immunologic features are associated with IgG4-related disease (IgG4-RD), the condition is defined as IgG4-related sclerosing mesenteritis (IgG4-RSM). ${ }^{3}$ Here, we report a case of a 70-yearold woman, who presented with a palpable intra-abdominal mass and vague abdominal pain, and was finally diagnosed with IgG4RSM based on histology and immunohistochemistry. This case report was approved by the Institutional Review Board (IRB) of Yonsei University Medical Center (IRB No. 4-2015-0650).

\section{CASE REPORT}

A 70-year-old woman presented with a palpable intra-abdominal mass and pain for about 3 months. She had a history of hypertension and a total hysterectomy 10 years earlier. Radiologically, a $7.9 \mathrm{~cm}$ lobulated mass was found in her right lower abdomen with multiple calcifications (Fig. 1A), suggesting a mesenteric-origin tumor. Positron emission tomography-computed tomography revealed a mesenteric mass with calcification in the rihgt lower quadrant of abdomen which had intense fluorodeoxyglucose (FDG) uptake. A few satellite nodules were also present in the mesentery; however, no other abnormal FDG uptake was identified. The patient underwent segmental resection of the small bowel. Grossly, the mucosal surface was intact and a multilobulated mass with whitish fibrosis was noted in the mes-

Corresponding Author

Sang Kyum Kim, MD, PhD

Department of Pathology, Yonsei University Medical Center, 50-1 Yonsei-ro, Seodaemun-gu, Seoul 03722, Korea

Tel: +82-2-2228-6751, Fax: +82-2-2227-7939, E-mail: nicekyumi@yuhs.ac

Received: October 6, 2015 Revised: November 27, 2015

Accepted: December 3, 2015 entery (Fig. 1B). Light microscopy revealed fat necrosis and a sclerosing fibrotic lesion including lymphocytic aggregation with germinal center formation and spindle cell proliferation (Fig. 2A). The spindle cells had abundant cytoplasm, a vesicular nucleus, and prominent nucleoli (Fig. 2B). In the periphery, acute inflammatory cells and eosinophils were seen. Chronic inflammatory cell infiltration into the venular wall with obliteration of the lumen juxtaposed to an artery was also seen (Fig. 2C).

As a result, three histologic findings of storiform pattern of fibrosis, lymphoplasmacytic infiltration, and obliterative phlebitis were distinct features of the presented case. Based on these findings, the differential diagnoses included inflammatory malignant fibrous histiocytoma, inflammatory myofibroblastic tumor, and SM. We performed immunohistochemical staining using anaplastic lymphoma kinase (ALK, D5F3), smooth muscle actin (SMA), Ki-67, IgG, and IgG4 antibodies. The overall Ki67 labeling index was low and was increased only in lymphoid follicles (Fig. 3A). ALK staining was negative and SMA staining revealed vascular obliteration (data not shown). Most of the plasma cells expressed IgG (Fig. 3B) and IgG4 (Fig. 3C), and the IgG4-positive cells/IgG-positive cells ratio was higher than $90 \%$. Therefore, we concluded the lesion as IgG4-RSM. Immediately after the surgery, blood samples were taken to measure the patient's levels of IgG and IgG4. IgG4 level has greatly increased $(2,130.0 \mathrm{mg} / \mathrm{L}$; reference range, 39.2 to $864.0 \mathrm{mg} / \mathrm{L})$, while $\mathrm{IgG}$ stayed within the normal range $(1,318 \mathrm{mg} / \mathrm{dL}$; reference range, 700 to $1,600 \mathrm{mg} / \mathrm{dL}$ ). The patient was discharged without complication, received a clinical work-up for IgG4-RSM in an outpatient department, and started on steroid therapy.

\section{DISCUSSION}

$\mathrm{SM}$ is a rare benign condition that presents with fibrosis, inflammation, and fat necrosis and occurs idiopathically in the 
small bowel mesentery. ${ }^{2}$ Clinically, patients with SM mostly complain of chronic severe pain and chronic non-specific problems such as nausea, vomiting, diarrhea, cramping, weight loss, and fever. ${ }^{4}$ Histologically, SM shows fibrosis with fat necrosis, chronic inflammation especially around the vessels, and variable focal calcification. In particular, if the immunologic and histologic characteristics are consistent with the IgG4-RD, it is classified as IgG4-RSM. ${ }^{3}$ The histologic features of IgG4-RSM include more than two of the followings: lymphoplasmacytic infiltration, storiform fibrosis, and obliterative phlebitis. The immunologic criterion is IgG4-positive/IgG-positive cells $>40 \%$, but this ratio is considered secondary in importance to the histological appearance.

Kerdsirichairat $e t ~ a l .^{3}$ conducted a systematic literature review
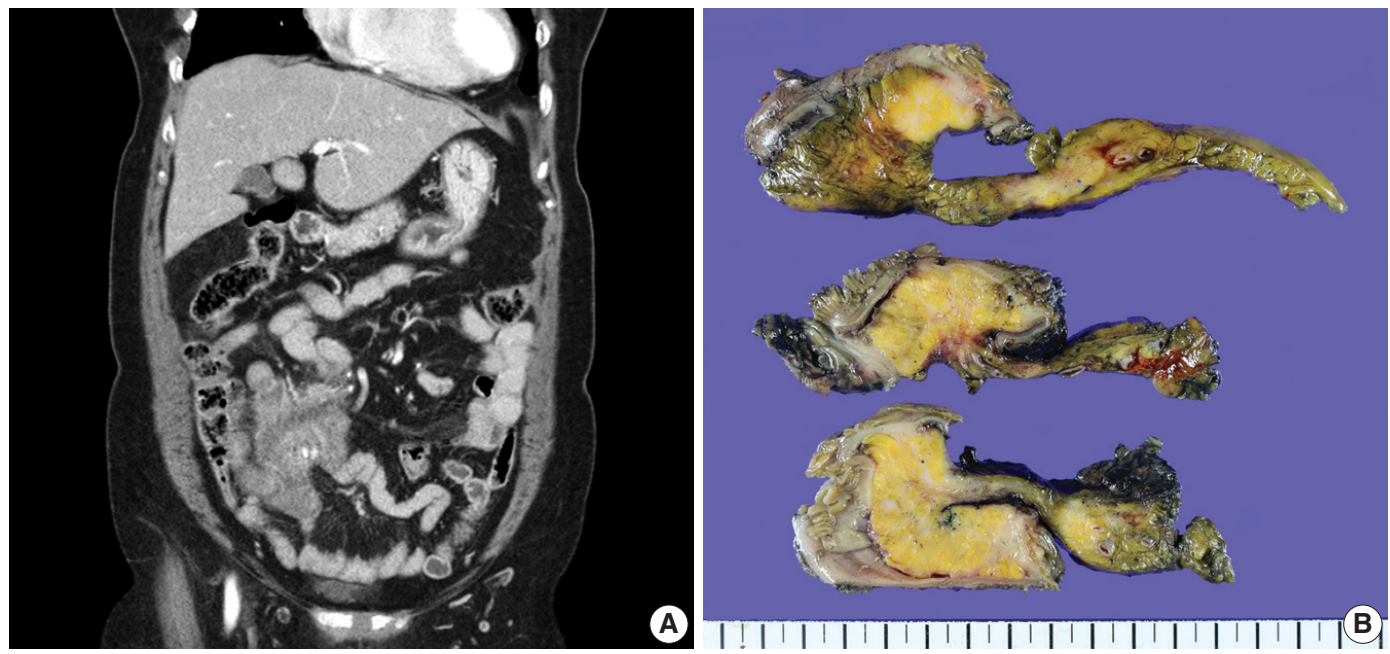

Fig. 1. (A) A $7.9 \mathrm{~cm}$ lobulated mass with multiple variable calcifications is seen in the right lower abdomen on abdominal computed tomography. (B) An ill-defined whitish fibrotic lesion is noted in the mesentery.
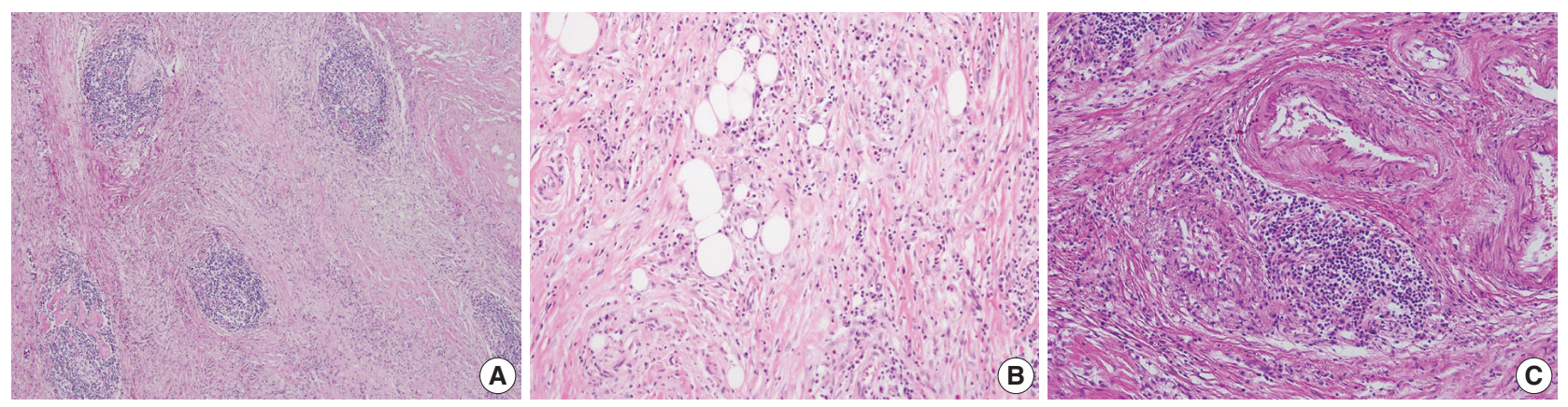

Fig. 2. (A, B) The microscopic findings show fat necrosis and a sclerosing fibrotic lesion (storiform fibrosis) with lymphocytic aggregation within the germinal center formation and spindle cell proliferation. (C) Obliterative phlebitis just next to an artery is also seen.
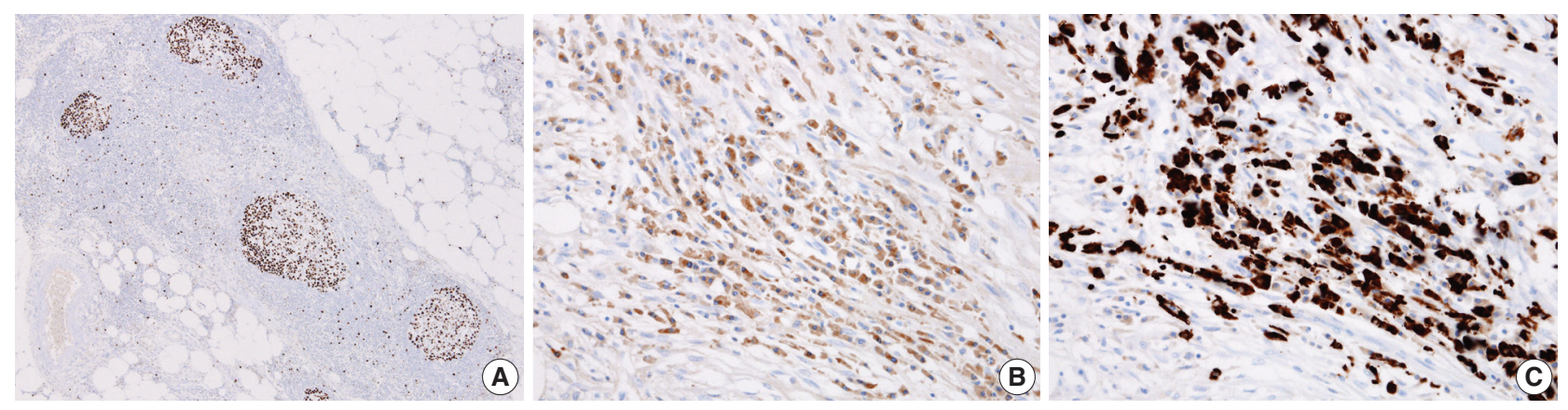

Fig. 3. (A) A low Ki-67-labeling index is shown with increase only in the lymphoid follicle. The lgG4-positive cells (C)/lgG-positive cells (B) ratio is higher than $90 \%$. 
of SM with IgG4 testing, and 11 out of 18 cases in total met the criteria for IgG4-RSM. The age of patients with IgG4-RSM ranges from 48 to 82. IgG4-RSM has a male predominance similar to SM. IgG4-RSM is known as a rare mesenteric disease of unknown etiology, although prior abdominal surgery can result in SM. Emory et al. ${ }^{2}$ reviewed 84 cases of SM and a history of trauma or surgery was present in four of 84 patients. Two of four patients had hysterectomies 20 and 12 years earlier. Our patient has a history of total abdominal hysterectomy, which might be the cause of the IgG4-RSM.

There is no generalized consensus regarding the treatment of SM, including medical therapy, surgical therapy, and surgery with additional medical therapy. In one study, the response rates were shown to be good for all therapies $(65 \%, 82 \%$, and $71 \%$, respectively), ${ }^{3}$ but the limited number of cases made it difficult to determine a treatment of choice. Even, when patients with SM are not treated, they show a high rate of spontaneous resolution. ${ }^{5}$ Therefore, therapy is needed when a patient displays symptoms.

In our case, the patient's differential diagnoses included inflammatory malignant fibrous histiocytoma and inflammatory myofibroblastic tumor, based on microscopic features. Inflammatory malignant fibrous histiocytoma shows neoplastic histiocyte-like cells mixed with neutrophils and other inflammatory cells. It has a high Ki-67 index, but in our patient's case, the overall Ki-67 index was low and was only increased in lymphoid follicles. Inflammatory myofibroblastic tumor shows spindle cell proliferation with myofibroblastic differentiation, a collagen stroma, and chronic inflammatory cell infiltrations. However, it rarely shows obliterative phlebitis. ${ }^{6}$ It is immunoreactive for ALK in about half the cases and stains for SMA ${ }^{7,8}$ Also, the ratio of IgG4-positive/IgG-positive cells is markedly lower than that of IgG4-RD.? In our case, SMA staining was non-specific and only confirmed the obliterative phlebitis. The high ratio of IgG4-positive/IgG-positive cells also supported IgG4-RSM.

In summary, we present a case of IgG4-RSM, which is compatible with previously described cases. The condition is benign and has a self-limiting course, but the pathologic diagnosis is important to exclude malignant conditions, which can be mis- diagnosed on radiologic analysis. The rarity of the disease limits its clear characterization, and more cases and studies are needed.

\section{Conflicts of Interest}

No potential conflict of interest relevant to this article was reported.

\section{REFERENCES}

1. Sulla JV. Mesenterite e sclerosante. Policlinico Prat 1924; 31: 575-81.

2. Emory TS, Monihan JM, Carr NJ, Sobin LH. Sclerosing mesenteritis, mesenteric panniculitis and mesenteric lipodystrophy: a single entity? Am J Surg Pathol 1997; 21: 392-8.

3. Kerdsirichairat T, Mesa H, Abraham J, et al. Sclerosing mesenteritis and IgG4-related mesenteritis: case series and a systematic review of natural history and response to treatments. Immunogastroenterology 2013; 2: 119-28.

4. Parra-Davila E, McKenney MG, Sleeman D, et al. Mesenteric panniculitis: case report and literature review. Am Surg 1998; 64: 768-71.

5. Akram S, Pardi DS, Schaffner JA, Smyrk TC. Sclerosing mesenteritis: clinical features, treatment, and outcome in ninety-two patients. Clin Gastroenterol Hepatol 2007; 5: 589-96.

6. Minato H, Shimizu J, Arano Y, et al. IgG4-related sclerosing mesenteritis: a rare mesenteric disease of unknown etiology. Pathol Int 2012; 62: 281-6.

7. Cessna MH, Zhou H, Sanger WG, et al. Expression of ALK1 and p80 in inflammatory myofibroblastic tumor and its mesenchymal mimics: a study of 135 cases. Mod Pathol 2002; 15: 931-8.

8. Qiu X, Montgomery E, Sun B. Inflammatory myofibroblastic tumor and low-grade myofibroblastic sarcoma: a comparative study of clinicopathologic features and further observations on the immunohistochemical profile of myofibroblasts. Hum Pathol 2008; 39: 846-56.

9. Yamamoto H, Yamaguchi H, Aishima S, et al. Inflammatory myofibroblastic tumor versus IgG4-related sclerosing disease and inflammatory pseudotumor: a comparative clinicopathologic study. Am J Surg Pathol 2009; 33: 1330-40. 